$17,641(5,120-42,895) \quad(p=0.002))$. There was no statistical difference in the non-POD group.

Transferrin was significantly lower in POD patients with HE $(n=143)$ compared to those that never developed HE $(n=93) \quad(1.420 \quad(1.100-1.720) \quad$ vs. $1.670 \quad(1.400-1.990)$ $(p<0.001))$. Levels were also significantly lower in the nonPOD group $(1.500(1.085-1.945)$ vs. 1.985 (1.445-2.365) $(\mathrm{p}=0.007))$.

Ferritin was significantly higher in POD patients with $\mathrm{HE}$ $(n=146)$ compared to those who never developed HE $(n=99)$. $(24,828 \quad(9,267-61,214) \quad$ vs. $17,149 \quad(3,153-37,994)$ $(p=0.009))$. There was no difference in the non-POD group.

No significant results were found when comparing circulating iron levels and transferrin saturations.

ROC curve analysis, however, showed that iron studies are poor at predicting survival in ALF. For example the area under the curve for transferrin to predict survival in POD patients was 0.662 . To predict encephalopathy free course of disease in the same group of patients was 0.666 .

Conclusions Iron indices, specifically ferritin and transferrin, are significantly different in patients with ALF and relate to aetiology and outcome. However they are poor at predicting survival.

\section{PTU-025 OBESITY IS ASSOCIATED WITH SEVERITY AND OUTCOME OF ALCOHOLIC HEPATITIS}

${ }^{1}$ Prebashan Moodley*, ${ }^{1,2}$ Ashwin Dhanda. 'University Hospitals Plymouth NHS Trust, Plymouth, UK; ${ }^{2}$ University of Plymouth, Plymouth, UK

\subsection{6/gutjnl-2019-BSGAbstracts.234}

Introduction Both sarcopenia and adiposity can influence the prognosis of patients with chronic liver disease. However, little is known of their interaction in patients with alcoholic hepatitis (AH). With the changing demographics of gender, age and body shape of $\mathrm{AH}$ patients, it is important to understand how obesity and sarcopenia affect AH severity and outcome. We aimed to determine whether quantity of skeletal muscle and adipose tissue influenced the outcome and severity of $\mathrm{AH}$ patients.

Methods We studied a prospective single centre cohort of patients with severe $\mathrm{AH}$, defined as recent onset jaundice (bilirubin $>80 \mu \mathrm{mol} / \mathrm{L}$ ) in heavy alcohol drinkers (>80g etha$\mathrm{nol} /$ day in males; $>60 \mathrm{~g}$ in females), AST:ALT $>2$ and discriminant function $(\mathrm{DF})>32$. Patients received standard medical care including 28 days of corticosteroids in the absence of active infection. Clinical and laboratory parameters were obtained at baseline and patient survival recorded at 6 months. CT scans from patients taken within 3 months of their index presentation were evaluated for adipose tissue and skeletal muscle area. An image at the level of L3/4 vertebrae was analysed using Image J software according to published methodology (Gomez-Perez, JPEN 2016) and total abdominal, intra-peritoneal and skeletal muscle areas were measured.

Results Of a total 28 patients, 21 (mean age 49, DF 74, MELD 19; 11 females) had an eligible CT scan and were included in the final analysis. There were 5 deaths within 6 months (2 females). Total abdominal area was higher in nonsurvivors vs survivors at 6 months $\left(1010 \mathrm{v} 715 \mathrm{~cm}^{2}\right.$; $\mathrm{p}=0.003)$ and correlated with severity of $\mathrm{AH}$ measured by MELD $(r=0.50 ; p=0.04)$. Waist circumference and adipose tissue area were higher in non-survivors than survivors at 6 months $\left(126 \mathrm{v} 105 \mathrm{~cm} ; \mathrm{p}=0.02\right.$ and $576 \mathrm{v} 406 \mathrm{~cm}^{2}$; $\mathrm{p}=0.05$, respectively). Skeletal muscle area was no different between non-survivors and survivors (141 v $121 \mathrm{~cm}^{2}$; $\mathrm{p}=0.37)$. Multivariate analysis with total abdominal area, age, bilirubin, albumin, urea and MELD as co-variates showed that total abdominal area is an independent predictor of 6-month outcome in this dataset $(p=0.003)$.

Conclusions These data demonstrate that adiposity but not sarcopenia is associated with severity and predicts outcome of $\mathrm{AH}$. Obesity should be recognised as a risk factor in patients with $\mathrm{AH}$. Greater understanding of the interaction between alcohol and adipose tissue in AH may reveal new targets for treatment.

\section{PTU-026 EVIDENCE OF GUT-LIVER CROSSTALK AND GUT HOMEOSTASIS IN PARACETAMOL TOXICITY:IN VITRO STUDY USING IMPEDANCE-BASED ASSAY}

${ }^{1}$ Katie Morgan*, ${ }^{2}$ Kay Samuel, ${ }^{1}$ Wesam Gamal, ${ }^{1}$ Steve Morley, ${ }^{1}$ Peter Hayes, ${ }^{3}$ Pierre Bagnaninchi, ${ }^{1}$ John Plevris. ${ }^{1}$ The University Of Edinburgh, Edinburgh, UK; ${ }^{2}$ Scottish National Blood Transfusion Service, Edinburgh, UK; ${ }^{3} \mathrm{MRC}$ Centre for Regenerative Medicine, Edinburgh, UK

\subsection{6/gutjnl-2019-BSGAbstracts.235}

Introduction Drug induced liver injury accounts for approximately one half of all acute liver failure cases, with Paracetamol (APAP) being one of the most reported hepatotoxins [1]. We used an in vitro approach to model APAP transport from gut to liver where metabolic products (NAPQI) can cause hepatocellular damage. Investigating the mechanisms of a gut-liver axis in vitro may contribute to a better understanding of APAP toxicity.

Methods The cellular impedance ECIS Z $\Theta$ platform was used to study the gut-liver axis and effects of APAP. Caco-2 and HepaRGs were seeded on separate $8 \mathrm{~W} 10+\mathrm{E}$ ibidi arrays. The Caco-2 array was collagen coated. Step 1: Previously established concentrations of APAP [2] were applied to confluent Caco-2s (day 10), and impedance monitored for 24 hrs. Step 2: This 'preconditioned' culture medium was then transferred to HepaRGs (day 11) for 24 hrs. Step 3: preconditioned culture medium was then transferred from HepaRGs to fresh Caco-2s (day 12) for 24 hrs.

Results Step 1: using an impedance based cellular assay, we show a tightening of barrier function in Caco-2s treated with $20 \mathrm{mM}$ APAP. Step 2: only the highest concentration $(20 \mathrm{mM}$ APAP) shows substantial loss of impedance on hepatocytes. Step 3: we demonstrate that barrier function has become sensitized on Caco-2s with an increase in impedance at $10 \mathrm{mM}$ Discussion and Conclusions This study demonstrates a potential cross-talk between hepatocytes and enterocytes and reveals a homeostatic effect of gut barrier function in presence of APAP. Exposing hepatocytes to toxic levels of APAP taken from Caco-2's attenuates toxicity at 5 and $10 \mathrm{mM}$ concentrations compared to previous study [2] where a dose-dependent loss of tight junctions was observed (5-20 mM APAP). Further exposure of preconditioned media on fresh Caco-2s (step 3) shows that tightening of barrier function is now achieved with lower APAP concentration $(10 \mathrm{mM})$ to reduce absorption of APAP. It is possible a liver-gut axis regulates APAP absorption through paracrine signals, though more work is needed. This may differ between population phenotypes and potentially be responsible for variability of toxic response in patients with APAP overdose. 